\title{
Robust cyclic berth planning of container vessels
}

\author{
Maarten Hendriks • Marco Laumanns • \\ Erjen Lefeber · Jan Tijmen Udding
}

Published online: 7 March 2010

(C) The Author(s) 2010. This article is published with open access at Springerlink.com

\begin{abstract}
We consider a container terminal operator who faces the problem of constructing a cyclic berth plan. Such a plan defines the arrival and departure times of each cyclically calling vessel on a terminal, taking into account the expected number of containers to be handled and the necessary quay and crane capacity to do so. Conventional berth planning methods ignore the fact that, in practice, container terminal operator and shipping line agree upon an arrival window rather than an arrival time: if a vessel arrives within that window then a certain vessel productivity and hence departure time is guaranteed. The contributions of this paper are twofold. We not only minimize the peak loading of quay cranes in a port, but also explicitly take into account the arrival window agreements between the terminal operator and shipping lines. We present a robust optimization model for cyclic berth planning. Computational results on a real-world scenario for a container terminal in Antwerp show that the robust
\end{abstract}

\footnotetext{
M. Hendriks · E. Lefeber $(\varangle) \cdot$ J. T. Udding

Systems Engineering Group, Department of Mechanical Engineering,

Eindhoven University of Technology, Den Dolech 2, P.O. Box 513,

5600 MB Eindhoven, The Netherlands

e-mail: A.A.J.Lefeber@tue.nl
}

M. Hendriks

e-mail: M.P.M.Hendriks@tue.nl

J. T. Udding

e-mail: J.T.Udding@tue.nl

M. Laumanns

Institute for Operations Research, ETH Zurich, 8092 Zurich, Switzerland

e-mail: Laumanns@ifor.math.ethz.ch

J. T. Udding

PSA HNN, Napelsstraat 79, 2000 Antwerp, Belgium

e-mail: JanTijmen.Udding@psahnn.be 
planning model can reach a substantial reduction in the crane capacity that is necessary to meet the window arrival agreements, as compared to a deterministic planning approach.

Keywords Container operations $\cdot$ Berth planning $\cdot$ Robustness $\cdot$ Linear programming

\section{Introduction}

Over the last decades, air, road, rail and sea transportation of people and goods has grown tremendously. Since the physical expansion of transportation systems is commonly very expensive and sometimes impossible, the existing infrastructure has to be operated very efficiently to cope with the enlarged utilization. Timetables become more dense and with that more vulnerable to stochastic disturbances. In poorly constructed timetables, the delay of a single transportation object might propagate through the entire schedule making it very difficult or even impossible to recover. To deal with stochastic disturbances in dense transportation schedules, two (complementary) approaches are gaining more and more attention (Clausen 2007): (1) pro-active robustness, which builds in buffer times and other characteristics into strategic or tactical timetables to absorb disturbances and thus to prevent delay propagation through a schedule, and (2) disruption management, which is concerned with operational recovery after a disruption.

The research in this paper focuses on incorporating pro-active robustness in a cyclic nominal berth plan for container vessels. We consider a terminal operator, who provides a number of vessel lines with the facility logistics of discharging, loading, transporting and storing containers. Each vessel line owns a vessel fleet to maintain several repetitive loops along ports all over the world. Commonly, the number and phasing of the vessels of one loop are such that they call on each port cyclically (typically once a week). The current policy of most terminal operators is to adopt each customer's (vessel line's) preferred arrival and departure times. This results in a so-called nominal berth plan, which presents the nominal arrival and departure time of each of the vessel lines. The time interval between the nominal arrival and departure time is the nominal vessel process time. Note that besides this nominal berth plan at a strategic level, the terminal operator also makes at an operational level a berth allocation in which actual quay space is assigned to vessels. The latter is not subject of this study.

Due to all kinds of events during travel (e.g., tailwind, storms, technical problems), container vessels might arrive earlier or later than their nominal arrival time. Therefore, the terminal operator and each of the vessel lines agree upon an arrival window, which is placed around the nominal arrival time. Two kinds of arrivals can be distinguished: arrivals (1) within, and (2) outside the window. If a vessel arrives within its window, the terminal operator guarantees to process this vessel within the nominal vessel process time. If a vessel arrives outside its window, the terminal operator is not bound to any guaranteed process time. Nevertheless, he aims to serve the vessel as soon as possible, but without jeopardizing the process time agreements for other vessels. Though this time window is crucial for operations, it is not often taken into 
account when constructing a plan. The goal of this paper is to develop a planning model that takes this arrival window into account explicitly.

A plan traditionally constructed to accommodate each vessel line's preferred arrival and departure times (neglecting the agreed arrival windows) might for particular arrival scenarios within the windows, (1) not yield a feasible operational plan due to lack of quay meters or (2) require a large amount of crane capacity to fulfill the process time agreements. We are therefore interested in robustness improvements when a berth plan, constructed in the traditional way, can be slightly modified by shifting the arrival times (windows). In our definition, a berth plan is robust with respect to a given set of arrival windows if a feasible solution exists for each arrival scenario where all vessels arrive within their windows. The price of this robustness (Bertsimas and Sim 2004) is the additionally required crane capacity to achieve this, compared to the necessary crane capacity of the nominal problem with deterministic arrival times. The problem is hence to develop a window-based method that minimizes the maximally required crane capacity for all scenarios where vessels arrive within their arrival windows.

We develop a robust mixed integer linear program (MILP) model, which explicitly incorporates the process time agreements and minimizes the maximally required crane capacity reservation. Besides the nominal arrival times of vessels being decision variables, the model also considers time-variant crane capacity reservations per vessel to be decision variables. This means that the number of cranes processing a vessel may vary from one time slot to the next. The model thus incorporates two degrees of freedom: (1) shifting the nominal arrival times of vessel lines and (2) reserving a timevariant crane capacity for each vessel. The goal is to exploit these decisions to better balance the workload over time and hence to minimize the maximal crane capacity reservation ever required. We perform a case study using a representative nominal berth plan as well as other representative problem data from the terminal operator PSA HNN in Antwerp, Belgium. Computational results of the window-based method demonstrate that with only small modifications to the nominal arrival times in this berth plan a significant reduction in the maximal crane capacity reservation can be obtained.

Conventional berth planning and allocation methods (Cordeau et al. 2005; Hansen et al. 2007; Imai et al. 2001, 2005, 2007; Kim and Moon 2003; Lim 1998; Nishimura et al. 2001; Park and Kim 2003; Wang and Lim 2007) ignore stochastic arrivals while constructing a nominal berth plan or nominal berth allocation. As a consequence, disturbed arrivals that are still within the arrival windows may put a high pressure on quay and crane capacities in order to meet the process time agreements. Our proposed planning model enables us to construct a nominal berth plan in the conventional fashion as a special case, by setting the arrival window width to zero. Hence, we can compare a berth plan found by the window-based planning method with a berth plan constructed in the conventional fashion. As expected, the window-based plan requires a slightly larger maximal crane capacity than the conventional plan if actual disturbed arrivals turn out to be close to the nominal arrival times. However, the window-based plan requires a significantly smaller maximal crane capacity if actual arrivals are more disturbed, but are still well within the arrival windows.

After a brief review of robustness in transportation planning in Sect. 2, the problem at hand is formally phrased in Sect. 3. Moreover, a robust MILP model is developed 
to construct a window-based plan that minimizes the maximal crane capacity reservation ever required. In Sect. 4, results of a case study show that with only small modifications to the nominal arrival times, already the cost of the robust solution can be reduced significantly. In a second experiment, the performance of both the windowbased plan and a nominal plan constructed in the conventional fashion, are compared. As expected, the window-based plan requires a slightly larger maximal crane capacity reservation for small deviations from the found nominal arrival times. However, for more disturbed arrivals that are still well within the arrival window, the window-based plan requires a significantly smaller maximal crane capacity than the plan constructed in the conventional fashion. We end with conclusions in Sect. 5.

\section{Related work}

The incorporation of pro-active robustness into transportation planning models has become increasingly prominent, in particular in airline operations planning, where Clausen (2007) as well as Ball et al. (2007) address a number of robustness ideas. Of particular interest for the problem considered in this paper is the approach of adding slack between connecting flights in Lan et al. (2006). Flight schedules are often that tight that in case of a small plane delay, passengers might miss their connecting flight. Adding more slack between the flights is beneficial for the passengers but reduces the productivity of the airline fleet. The authors propose an MILP in which both a flight's arrival time and the departure time of its connecting flight(s) can be scheduled somewhere within a window. Each possible arc between a time slot in the arrival window and a time slot in the departure window is called a copy. Each copy implies a connecting travel time and, as determined from historical data, induces a probability of passengers missing their connecting flight (if the travel time exceeds the connecting time). Given a set of flights within a restricted amount of time, the objective is to select exactly one copy for each pair of connecting flights such that the expected total number of delayed passengers is minimized.

With respect to pro-active robustness in railway applications, a few approaches can be found (Caimi et al. 2007; Vromans et al. 2007). The authors in Vromans et al. (2007) consider a stochastic optimization model for building in time buffers between connecting trips based on arrival and departure distributions for each train. They propose a model, which allocates a restricted amount of time supplement to a number of trips to minimize the expected total amount of delay. Experimental results show that slightly modifying the existing timetable can reduce the average passenger delay substantially. The study in Caimi et al. (2007) embeds robustness into the train timetable by allocating time windows for arrival and departures rather than single arrival and departure times. The model is stated as a flexible cyclic event scheduling problem, which guarantees that any particular choice of event times within the computed intervals is feasible. The resulting window-based timetable is therefore robust to disturbances within these bounds. The total sum of interval lengths can be traded off against the total sum of travel times in a bi-objective optimization formulation.

To the best of our knowledge, only one study (Moorthy and Teo 2006) addresses a stochastic berth allocation problem in a container terminal. The authors propose a 
sequence pair approach to assign vessels in time and space given arrival distributions and preferred berth positions. Conflicting objectives are to minimize the total expected delay and the deviations from preferred berth locations. Once a cyclic nominal berth allocation is determined, simulations with stochastic arrivals are performed. Results are compared with those of a model that ignores disturbances. They show that taking disturbances into consideration yields a reduction in total delay on the operational level. One of the recommendations of the authors is to incorporate crane allocations, since it affects the port stay of vessels and therefore might have a significant impact on the robustness of a berth plan.

In this paper, we also aim for embedding robustness into an existing nominal berth plan. Although we guarantee sufficient quay and crane capacities for all arrival scenarios within the arrival windows, the actual berth allocation problem might be infeasible, e.g., due to fragmented space. From practice we know that using conservative estimations for vessel lengths and quay length avoids these problems. The actual berth location assignment is addressed in a subsequent study (Hendriks 2009). In contrast to the study in Moorthy and Teo (2006), our model does include the crane capacity allocation and therefore exhibits an additional flexibility to incorporate robustness. With this window-based planning tool, the nominal arrival times and crane capacity reservations are chosen such that the maximal crane capacity reservation is minimized while the process time agreements are still met.

\section{Quay crane capacity allocation model}

In this section, we derive a model for robust quay and crane capacity allocation under stochastic vessel arrivals. We consider a periodic setting, where a set of vessel lines $\mathcal{V}=\{1,2, \ldots, V\}$ has to be allocated required capacity (as measured in quay meters and crane capacity) over a set of discrete time slots $\mathcal{K}=\{1,2, \ldots, K\}$. Each vessel of vessel line $v \in \mathcal{V}$ has an associated length $M_{v}$ and an amount of containers $C_{v}$ to be discharged from and loaded onto it, which are given as fixed input data. The terminal has a total length of $L$. The goal is to find a feasible assignment of arrival and berth time slots (and associated crane capacity reservations) so that the maximal crane capacity over all arrival scenarios of a bounded uncertainty set is minimized (a min max problem). The problem is thus developed as a robust cyclic capacity assignment problem, and its corresponding formulation as a mixed-integer linear program (MILP) will be given.

\subsection{Vessel processing}

Dependent on the length $M_{v}$ of vessel $v$, a maximum number $S_{v}$ of quay cranes can process vessel $v$ simultaneously. In practice, quay cranes with different processing rates are present in the terminal. We do not take the specific allocation of quay cranes to vessels into account yet, but consider an average processing rate $\bar{\lambda} \in \mathbb{N}$ for all quay cranes. Dependent on the length of a vessel $v$ the cranes operate with a certain efficiency $\eta_{v} \in[0,1]$; the smaller the length, the lower the efficiency. 
Then the minimal handling time of vessel $v$ in terminal $t$ depends on (1) the mean processing rate $\bar{\lambda}$ in terminal $t,(2)$ the efficiency $\eta_{v}$ of quay cranes operating vessel $v$, (3) the maximal number of quay cranes that can process vessel $v$ simultaneously, and (4) the total number of containers $C_{v}$ to be discharged from and loaded onto vessel $v$. The processing time of vessel $v$ is assumed to be inversely proportional to the first three of these items and proportional to the latter. The minimal integer number of time slots $P_{v}^{\min }$ required to process vessel $v$ can thus be determined as follows:

$$
P_{v}^{\min }=\left\lceil\frac{C_{v}}{\eta_{v} S_{v} \bar{\lambda}}\right\rceil,
$$

where $\lceil x\rceil$, denotes the smallest integer not less than $x$.

\subsection{Arrival windows and process time agreements}

According to the agreements, the terminal operator has to process a vessel within its nominal process time $P_{v}^{\text {nom }}$ if and only if that vessel arrives within its arrival window. We assume the width $2 W_{v}$ of the arrival window for vessel $v$ to be equal to an integer number of time slots. In practice, the nominal vessel process time agreed upon by the vessel line of vessel $v$ and the terminal operator is a factor $\alpha_{v}$ larger than the minimal process time. This we approximate by

$$
P_{v}^{\text {nom }}=\left\lceil\alpha_{v} P_{v}^{\min }\right\rceil
$$

where $P_{v}^{\text {nom }}$ is the nominal number of time slots in which vessel $v$ has to be processed only if it arrives within its arrival window, and $\alpha_{v} \geq 1$. Commonly, the value of $\alpha_{v}$ is significantly larger than 1 , which implies that vessel $v$ does not have to be processed with the maximal number of cranes $S_{v}$ permanently while berthed. Of course a vessel may also be processed faster than $P_{v}^{\text {nom }}$ time slots. Furthermore, we assume $2 W_{v}+P_{v}^{\text {nom }}<<K$, i.e., the number of time slots a vessel might possibly be berthed is much smaller than the cycle length. The model parameters together with their definitions are summarized in Table 1.

Let $t_{v}$ denote the nominal arrival time of vessel $v$, and $l_{v}$ and $r_{v}$ denote respectively the left and right end of the arrival window of width $2 W_{v}$, i.e., $l_{v}=t_{v}-W_{v}$ and $r_{v}=t_{v}+W_{v}$ (modulo $K$ ). The principle of the process time arrival agreements for vessel $v$ is illustrated in the top of Fig. 1. If vessel $v$ arrives somewhere within its window (up to $W_{v}$ time slots early or late), the terminal operator has to process vessel $v$ no more than $P_{v}^{\text {nom }}$ time slots later.

\subsection{Robust optimization problem}

Based on the vessel processing model and arrival window concept described above, we now state the capacity allocation problem as a robust MILP. The goal is to improve an existing nominal berth plan provided by PSA HNN. The cost of an optimal robust solution is decreased by slightly modifying some nominal arrival times, and reserving 
Table 1 Model parameters

\begin{tabular}{ll}
\hline Parameter & Definition \\
\hline$V$ & Number of vessels in the set \\
$K$ & Number of discrete time slots in the cyclic plan \\
$L$ & Terminal quay length (m) \\
$M_{v}$ & Length of vessel $v(\mathrm{~m})$ \\
$C_{v}$ & Number of containers to be discharged from and loaded onto vessel $v$ \\
$S_{v}$ & Maximal number of quay cranes that can process vessel $v$ simultaneously \\
$\bar{\lambda}$ & Average crane rate (containers/time slot) \\
$\eta_{v}$ & Crane efficiency on vessel $v$ \\
$W_{v}$ & Maximal deviation (early or late) from nominal arrival time that is still \\
$P_{v}^{\min }$ & within the arrival window of vessel $v$ \\
$P_{v}^{\text {nom }}$ & Minimal process time of vessel $v$ \\
$\alpha_{v}$ & Nominal process time of vessel $v$ \\
\hline
\end{tabular}

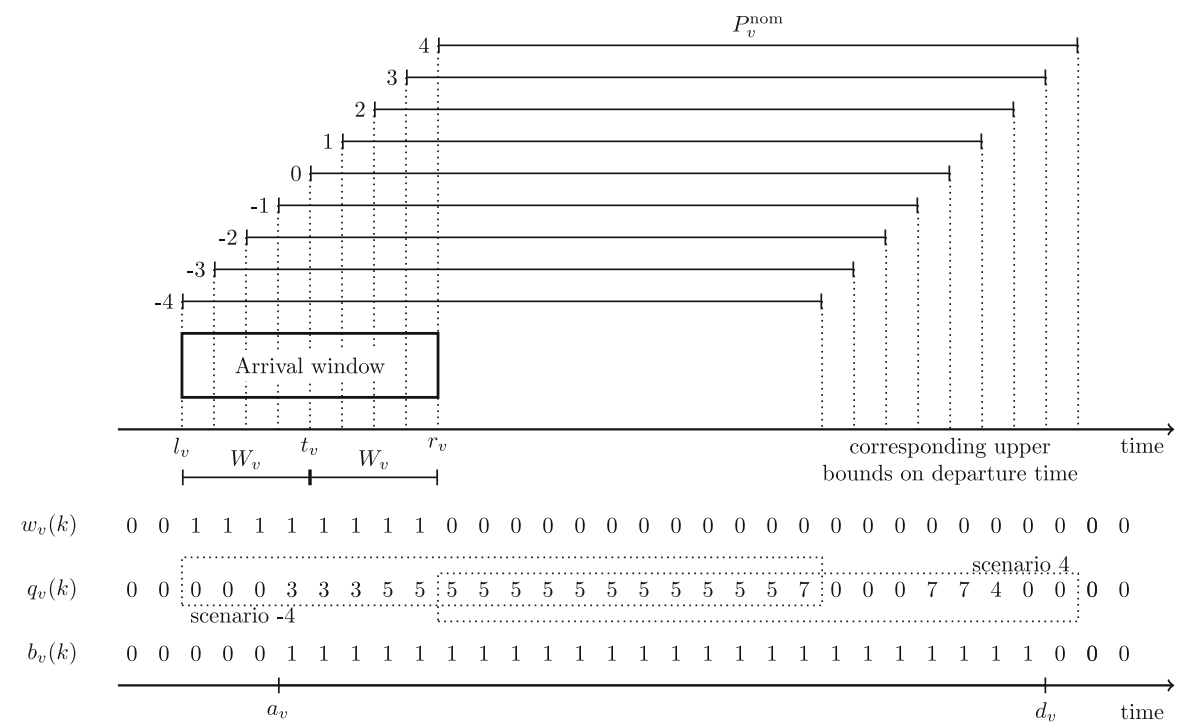

Fig. 1 Upper bound on the departure time for nine arrival scenarios within the window, i.e., $W_{v}=4$, where $P_{v}^{\text {nom }}=20$ (top). Relationship between $l_{v}, t_{v}, r_{v}, W_{v}, a_{v}$, and $d_{v}$ (bottom)

crane capacities to satisfy the agreements for all arrivals scenarios within the windows. While robustness of this berth plan in our definition is the property that the arrival agreements are satisfied for each of the arrival scenarios within the windows, we aim to achieve this robustness with minimum cost, that is, the crane capacity reservation required to achieve this should be minimized. For notational convenience, we choose the left end of the arrival window $l_{v}$ as a decision variable for constructing the nominal 
berth plan, rather than the nominal arrival time $t_{v}$. The second set of decision variables is the amount of crane capacity reserved for vessel $v$ during time slot $[k, k+1\rangle$, which we denote by $q_{v}(k)$. In addition to these primary decision variables, we introduce a set of auxiliary variables as follows:

$r_{v}=$ Right end of arrival window of vessel $v$.

$w_{v}(k)= \begin{cases}1 & \text { if time slot }[k, k+1\rangle \text { lies within the arrival window of vessel } v \\ 0 & \text { otherwise }\end{cases}$

$e_{v}^{w}= \begin{cases}1 & \text { if } l_{v}>r_{v} \\ 0 & \text { if } l_{v} \leq r_{v}\end{cases}$

$a_{v}=$ Earliest allowed berthing time for vessel $v$

$d_{v}=$ Latest allowed berthing time for vessel $v$

$b_{v}(k)= \begin{cases}1 & \text { if vessel } v \text { can possibly be berthed during time slot }[k, k+1\rangle, \\ 0 & \text { otherwise }\end{cases}$

$e_{v}= \begin{cases}1 & \text { if } a_{v}>d_{v} \\ 0 & \text { if } a_{v} \leq d_{v}\end{cases}$

$m_{v}(k)=$ Amount of quay meters reserved for vessel $v$ during time slot $[k, k+1\rangle$.

$Q=$ Available total crane capacity (to be minimized).

For vessel $v$ the arrival window can be positioned in time by positioning its left end $l_{v}$. From $l_{v}$ the values for $r_{v}$ and $w_{v}(k)$ should follow accordingly. First of all, we have to enforce that $w_{v}(k)=1$ if and only if time slot $[k, k+1\rangle$ lies between the left and right end of the window, i.e., between $l_{v}$ and $r_{v}$. Consider first the case that $l_{v} \leq r_{v}$. We need that $w_{v}(k)=0$ for $k \geq r_{v}$, or $k \cdot w_{v}(k) \leq r_{v}-1$. Furthermore we need that $w_{v}(k)=0$ for $k<l_{v}$, or $(K-k) \cdot w_{v}(k) \leq K-l_{v}$. By requiring that the sum of all $w_{v}(k)$ equals $r_{v}-l_{v}$ we have that $w_{v}(k)=1$ if and only if time slot $[k, k+1\rangle$ lies between the left and right end of the window. Similar reasoning for the case $l_{v}>r_{v}$ results in (see also Hendriks 2009):

$$
\begin{aligned}
& 1-l_{v} \leq k \cdot\left(w_{v}(k)-e_{v}^{w}\right) \leq r_{v}-1 \quad \forall v, k \\
& r_{v}-K \leq(K-k) \cdot\left(w_{v}(k)-e_{v}^{w}\right) \leq K-l_{v} \quad \forall v, k \\
& \sum_{k=1}^{K}\left(w_{v}(k)-e_{v}^{w}\right)=r_{v}-l_{v} \quad \forall v .
\end{aligned}
$$

In addition, the width of the arrival window for vessel $v$ is fixed to $2 W_{v}$. Considering the discrete time model, this implies that there have to be $2 W_{v}+1$ possible arrival time slots for vessel $v$ :

$$
\sum_{k=1}^{K} w_{v}(k)=2 W_{v}+1 \quad \forall v
$$


Similarly as in (3), (4), and (5) we can also enforce that $b_{v}(k)=1$ if and only if time slot $[k, k+1\rangle$ lies between $a_{v}$ and $d_{v}$.

$$
\begin{aligned}
& 1-a_{v} \leq k \cdot\left(b_{v}(k)-e_{v}\right) \leq d_{v}-1 \quad \forall v, k \\
& d_{v}-K \leq(K-k) \cdot\left(b_{v}(k)-e_{v}\right) \leq K-a_{v} \quad \forall v, k \\
& \sum_{k=1}^{K}\left(b_{v}(k)-e_{v}\right)=d_{v}-a_{v} \quad \forall v .
\end{aligned}
$$

We have to reserve quay meters and crane capacity for vessel $v$ only for those time slots $K$ where $b_{v}(k)=1$. We consider the planning problem at a strategic level, neglecting the integer nature of the actual berthing problem and crane assignment problem. This is dealt with at the tactical planning level. At this strategic level we only make sure that the terminal quay length $L$ is not exceeded, and we try to minimize the maximal crane capacity. Note that even when sufficient quay meters are available for an arriving vessel, it might not be able to berth due to fragmented space. From practice we know that using conservative parameters for vessel length and quay length, this problem is avoided.

If time slot $[k, k+1\rangle$ is an allowed berth time slot of vessel $v$, i.e., $b_{v}(k)=1, M_{v}$ quay meters have to be reserved during that time slot:

$$
m_{v}(k)=M_{v} \cdot b_{v}(k) \quad \forall v, k
$$

The sum of lengths of all vessels that are possibly berthed during time slot $[k, k+1\rangle$ should never exceed the terminal quay length $L$ :

$$
\sum_{v=1}^{V} m_{v}(k) \leq L \quad \forall k
$$

If time slot $[k, k+1\rangle$ is reserved for vessel $v$, the crane capacity reserved for vessel $v$ during that time slot is limited by the number of cranes that can process vessel $v$ simultaneously:

$$
q_{v}(k) \leq S_{v} \cdot b_{v}(k) \quad \forall v, k
$$

To generate a robust solution, we now have to enforce that the process time agreements for all vessels $v$ are satisfied for all arrival scenarios. The agreements state that if vessel $v$ arrives within its window, its process time has to be within the nominal process time $P_{v}^{\text {nom }}$. This requires matching the arrival and departure of a vessel (characterized by $l_{v}, t_{v}, r_{v}, W_{v}, w_{v}(k)$, and $P_{v}^{\text {nom }}$ ) with its reserved time for berthing (characterized by $b_{v}(k), a_{v}$, and $\left.d_{v}\right)$, as illustrated in the bottom part of Fig. 1.

Assume $W_{v}=4, P_{v}^{\text {nom }}=20$ and $C_{v}=80$. Then for each of the nine possible arrival scenarios we need to guarantee that a cumulative capacity of at least 80 is provided within the consecutive 20 time slots corresponding to that scenario, as indicated for scenarios -4 and 4 . Notice, however, that if in Fig. 1 the vessel arrives 
$4 \mathrm{~h}$ early (scenario -4$)$ it can not berth for the first $3 \mathrm{~h}$ yet $\left(b_{v}(k)=0\right.$; no quay has been reserved). This is no problem, as it still can leave after 20 time slots, as agreed between the terminal operator and the shipping line. Also when a vessel arrives $4 \mathrm{~h}$ late, enough capacity has been reserved such that vessel can leave in time (actually a little bit more than $2 \mathrm{~h}$ early). This actually holds for any of the nine possible arrival scenarios.

Hence, for each range of sequential time slots that starts from a time slot within the arrival window of vessel $v$ and ends $P_{v}^{\text {nom }}-1$ time slots later, the sum (over that particular range of sequential time slots) of reserved crane capacities should be sufficient to process at least $C_{v}$ containers. Since the time position of the arrival window of vessel $v$ is a decision variable on itself, we have to explicitly consider the sum of crane reservations for each possible range of sequential time slots of length $P_{v}^{\text {nom }}$ within the considered cycle. Only if the first time slot of such a range lies within the arrival window of vessel $v$, sufficient crane capacity for vessel $v$ during these time slots has to be reserved to process at least $C_{v}$ containers. To model this we make use of the value of the binary variable $w_{v}(k)$ :

$$
\sum_{i=k}^{k+P_{v}^{\mathrm{nom}}-1} \eta_{v} \bar{\lambda} \cdot q_{v}(i) \geq C_{v} \cdot w_{v}(k) \quad \forall v, k .
$$

Note that the cyclic nature of the system needs to be taken into account in (13) by replacing $q_{v}(i)$ with $q_{v}(i-K)$, whenever $i>K$.

Finally, the sum of reserved crane capacities of all vessels during time slot $[k, k+1\rangle$ should never exceed the available crane capacity $Q$ :

$$
\sum_{v=1}^{V} q_{v}(k) \leq Q \quad \forall k
$$

The objective is to minimize the available crane capacity $Q$ :

$$
\min _{l_{v}, q_{v}(k)} Q
$$

subject to the constraints (3) through (14), which guarantee that the capacity is sufficient over all arrival scenarios. Notice that minimizing the objective (15) is equivalent to minimizing the objective

$$
\min _{l_{v}, q_{v}(k)} \max _{k} \sum_{v=1}^{V} q_{v}(k) .
$$

\section{Case study}

The MILP proposed in the previous section determines a window-based plan with minimal crane capacity reservation necessary to process all vessels within their 
process time limits for all arrival scenarios within the considered bounds. Since the model explicitly incorporates the arrival window agreements, a plan is found which is robust to stochastic arrivals within the windows. In this section, we perform two experiments on a representative nominal berth plan constructed by PSA HNN. This nominal berth plan considers one terminal and fifteen vessel lines $(V=15)$, which all have one of their vessels calling exactly once each week. A time slot width of $1 \mathrm{~h}$ is chosen, so $K=168$. We set the arrival window width of vessel $v$ to $8 \mathrm{~h}$, so $W_{v}=4, \forall v$, which is a typical value in real-life container operations. This implies that if a vessel arrives up to $4 \mathrm{~h}$ earlier or $4 \mathrm{~h}$ later than its nominal arrival time, the terminal operator still has to process the vessel within its nominal process time $P_{v}^{\text {nom }}$. Furthermore, we assume a typical value of $\alpha_{v}=1.4 \forall v$, i.e., the nominal process time is $40 \%$ larger than the absolute minimal time required for serving a vessel. Realistic values for the other parameters in Table 1 are provided by PSA HNN, but not revealed for confidentiality reasons.

Two experiments are performed on this nominal berth plan. In the first experiment, we investigate the benefit of modification, i.e., the reductions in maximal crane capacity reservation that can be achieved by modifying some of the nominal arrival times in the provided nominal berth plan. Results show that with small modifications, already significant reductions in the maximal crane capacity reservation can be achieved.

In the second experiment, the performance of the window-based plan is compared to a plan constructed in the conventional fashion. The conventional plan can be constructed by simply setting the arrival window width to zero $\left(W_{v}=0, \forall v\right)$ in the MILP. Optimizing the MILP then results in an optimal cyclic plan with a minimal crane capacity reservation for the nominal arrival times. We want to compare the performance of window-based plans with conventional plans. Like in the first experiment we only allow small modifications to some of the nominal arrival times in the berth plan provided by PSA HNN. Next, we construct a window-based plan and a conventional plan and compare their sensitivity to different arrival disturbances.

Results show that the window-based plan requires a slightly larger maximal crane capacity reservation if actual deviations turn out to be close to the constructed nominal arrival times. However, the window-based plan requires a significantly smaller maximal crane capacity reservation if the actual arrivals turn out to be more disturbed, but are still well within the arrival windows. This highlights the importance of taking those uncertainties into account during the planning stage that really matter during operations, and to take them into account with the right magnitude by a careful choice of the uncertainty sets.

\subsection{Benefit of plan modification}

It is not realistic to run the MILP proposed in the previous section where we can arbitrarily shift the arrival windows of ships around. As mentioned before, vessel lines have fixed routes and a preferred nominal arrival time in each port they call on. Negotiations have to point out whether vessel lines are willing to slightly modify their preferred nominal arrival time in a particular port. We are therefore interested in a possible reduction in the maximal crane capacity reservation if relatively small 


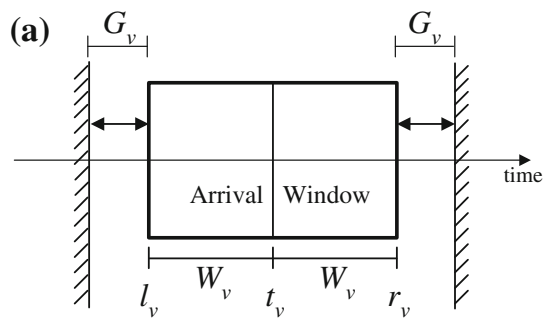

(b)

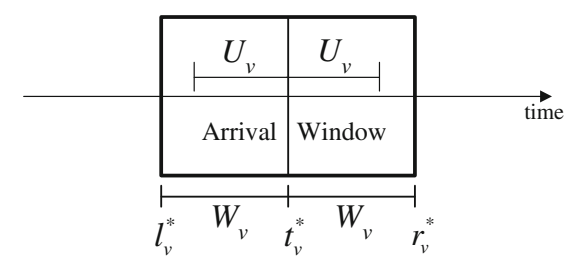

Fig. 2 Explanation of the parameters $G_{v}$ and $U_{v}$. a $G_{v}$ maximally allowed modification in the nominal arrival time $t_{v}$ of vessel $v, \forall v \in \mathcal{V}_{i}$, in the first experiment: benefit of plan modification. $\mathbf{b} U_{v}$ upperbound on deviations from nominal arrival time $t_{v}^{*}$ for vessel $v$ in the second experiment: windows-based plan versus conventional plan

Fig. 3 Robustness

improvements versus the level of allowed modification $G_{v}, v \in \mathcal{V}_{i}$

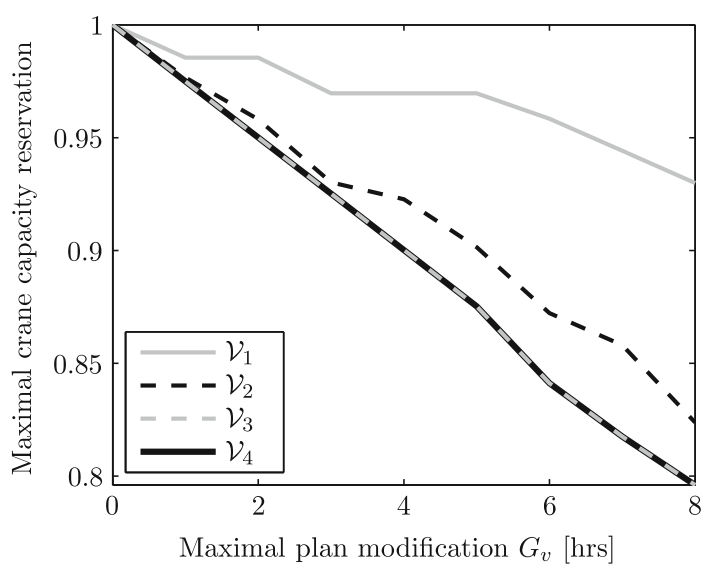

modifications to nominal arrival times in an existing berth plan are allowed. To that end, we add some constraints. Some of the vessels are not allowed to move, resulting in an additional equality constraint on $l_{v}$, whereas the arrival window of other vessels is allowed to move slightly (not more than $G_{v}$ hours), resulting in additional inequality constraints on $l_{v}$. To be more precise, the following sequence of four experiments is performed.

In experiment $i, i \in\{1,2,3,4\}$, a vessel subset $\mathcal{V}_{i}$ is arbitrarily selected from the representative data set provided by PSA HNN, where $\left|\mathcal{V}_{1}\right|=2,\left|\mathcal{V}_{2}\right|=4,\left|\mathcal{V}_{3}\right|=7$, $\left|\mathcal{V}_{4}\right|=V=15$, and $\mathcal{V}_{i} \subset \mathcal{V}_{i+1}, i \in\{1,2,3\}$. For each of the vessels in the subset $\mathcal{V}_{i}$ of experiment $i$, we allow a maximal modification of $G_{v}$ time slots to its provided nominal arrival time, by introducing appropriate upper and lower bounds on the arrival window position (see Fig. 2a). In each experiment $i \in\{1,2,3,4\}$, the window-based plan MILP with $W_{v}=4, \forall v$ is determined consecutively for $G_{v} \in\{0,1,2, \ldots, 8\}$, $v \in \mathcal{V}_{i}$. Results are depicted in Fig. 3. The (scaled) maximal crane capacity reservation is plotted versus the maximally allowed modification $G_{v}$ in the nominal arrival time of each $v$ in the selected vessel subset. Each curve in this plot depicts the outcome of one experiment $i$. 
In this figure, we notice the following:

- Each of the four curves is monotonically decreasing. This makes sense, since increasing the extent of maximal modification will never yield a higher amount of maximally required crane capacity reservation.

- Along the same line, we can explain that the curve of experiment $i+1$ never exceeds the curve of experiment $i$. Namely, if the plan of more vessels (actually if $\left.\left|\mathcal{V}_{i}\right|<\left|\mathcal{V}_{i+1}\right| \wedge \mathcal{V}_{i} \subset \mathcal{V}_{i+1}\right)$ is allowed to be modified, the maximal crane capacity reservation will never increase.

- One interesting result is that with allowing the nominal arrival time of seven vessels to be modified, the same improvements can be achieved as by allowing the plan of all fifteen vessels to be modified.

- Another interesting result is that by allowing the nominal arrival time of only four vessels to be modified, at least $75 \%$ of the total improvements can already be obtained.

- By modifying the nominal arrival time of four out of fifteen vessels maximally $2 \mathrm{~h}$, a reduction of about $5 \%$ in the maximal crane capacity reservation can already be achieved.

In summary, there appears to be a small number of vessels that are critical for reducing the worst-case peak load, and the model can help to identify these vessels together with the resulting potential capacity reduction.

\subsection{Window-based plan versus conventional plan}

By means of the previous experiment we showed that a slight modification of some of the nominal arrival times of vessels can result in a considerable reduction of the maximally required crane capacity. With our second experiment we want to illustrate the effect of explicitly taking into account the arrival window agreements. As mentioned before, the terminal operator and shipping lines agree on an arrival windows. Due to disturbances a vessel might arrive early or late. If a vessel arrives within its window, the terminal operator guarantees this vessel within the nominal vessel process time. The conventional approach to construct a nominal berth plan is to simply ignore the arrival window agreements and to determine the optimal deterministic berth plan. Such a conventional plan is constructed by setting the window width to zero, $W_{v}=0$, $\forall v$, and running the MILP.

We are interested in the performance of the window-based plan and the conventional plan for actual bounded arrivals, i.e., we assume the actual arrival deviation of vessel $v$ to be at most $U_{v}$, where we assume $0 \leq U_{v} \leq 4, \forall v$ (see Fig. 2b). So we first construct a plan (either window-based or conventional), fix the found nominal arrival times $t_{v}^{*}$ and then investigate the berth plan's sensitivity to bounded arrivals that are still within the arrival windows.

Subset $\mathcal{V}_{2}$ of the previous experiment is chosen since we think $\left|\mathcal{V}_{2}\right|=4$, is a reasonable guess for the number of vessels, whose nominal arrival times are allowed to be modified.

To determine the performance of the window-based plan and the conventional plan we use the following procedure for a particular value of $G_{v}, \forall v \in \mathcal{V}_{2}$, and subsequently evaluate their performances for a particular value of $U_{v}, \forall v$. 


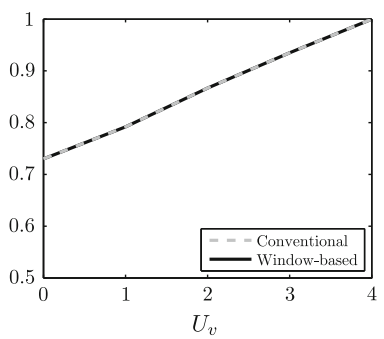

(a)

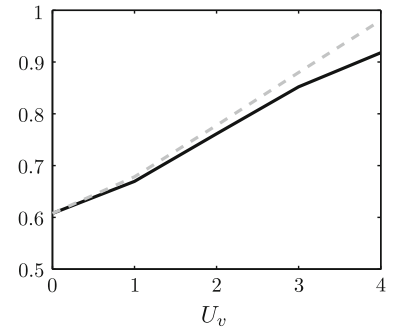

(b)

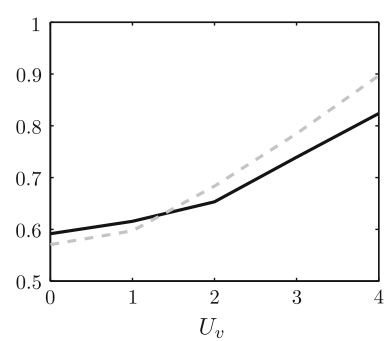

(c)

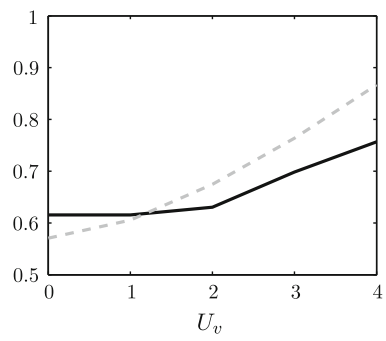

(d)

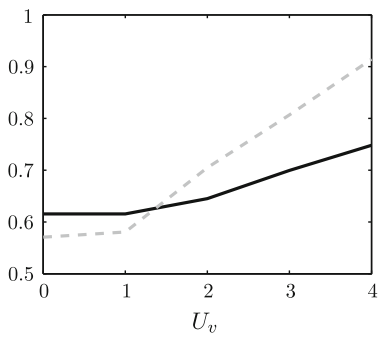

(e)

Fig. 4 Maximal crane capacity reservation versus $U_{v}$, for particular values of $G_{v}$. a $G_{v}=0$, b $G_{v}=4$, c $G_{v}=8, \mathbf{d} G_{v}=12, \mathbf{e} G_{v}=16$

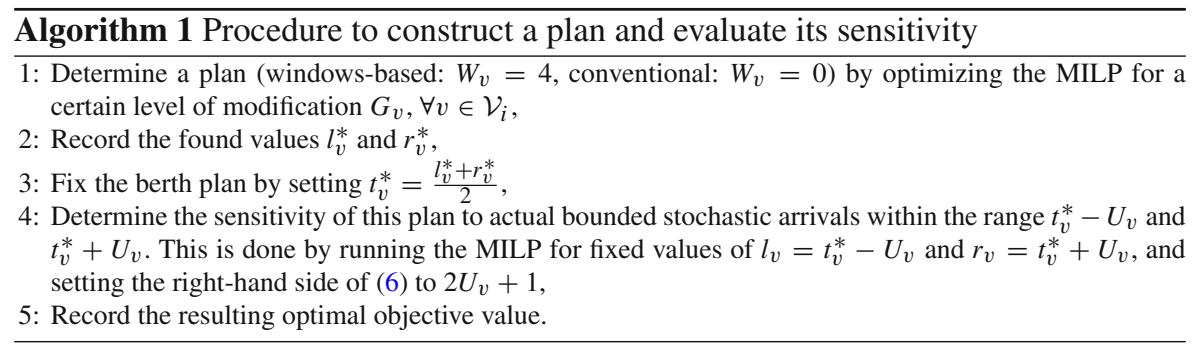

The performance (i.e., the maximal crane capacity reservation) of both the window-based plan and the conventional plan is evaluated on a grid of $G_{v}$ and $U_{v}$, where $G_{v} \in\{0,1,2, \ldots, 16\}, \forall v$, and $U_{v} \in\{0,1,2,3,4\}, \forall v$. Figure 4 depicts five projections of the maximally required crane capacity reservation as a function of $U_{v}$ for particular values of $G_{v}$. Figure 5 depicts five projections of the maximally required crane capacity reservation as a function of $G_{v}$ for particular values of $U_{v}$. In all figures, the values of the maximally required crane capacity reservation are scaled to the amount required for the case where $G_{v}=0$, and $U_{v}=4, \forall v$. So the value one represents the maximally required quay crane capacity reservation in the PSA HNN nominal berth plan, when the actual maximal arrival disturbances turn out to be the size of the arrival window, i.e., $U_{v}=W_{v}, \forall v$. Although both figures present the same data, some observations can be made much easier in Fig. 4, while others are better noticeable in Fig. 5. 


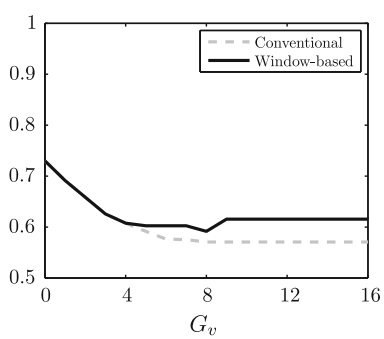

(a)

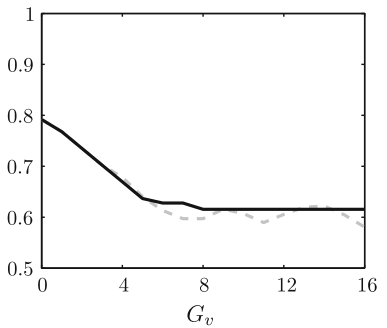

(b)

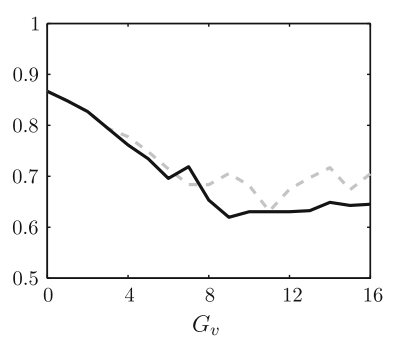

(c)

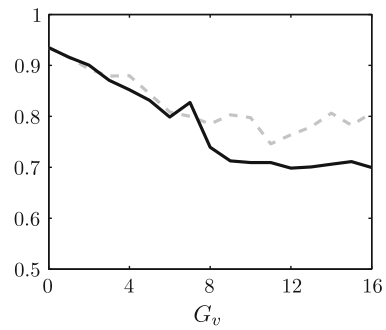

(d)

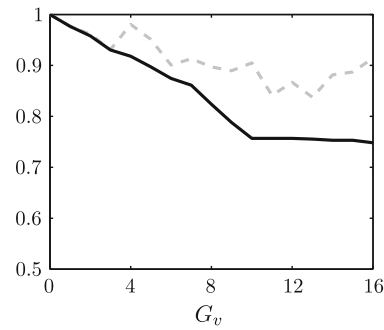

(e)

Fig. 5 Maximal crane capacity reservation versus $G_{v}$, for particular values of $U_{v}$. a $U_{v}=0, \mathbf{b} U_{v}=1$, $\mathbf{c} U_{v}=2, \mathbf{d} U_{v}=3$, e $U_{v}=4$,

From Fig. 4, we conclude the following:

- All curves in Fig. 4a through e are monotonically increasing. This is to be expected, since if the actual, maximal deviation $U_{v}$ from the constructed nominal arrival times increases, the maximal crane capacity reservation will never decrease.

- The two curves in Fig. 4a are identical. This is to be expected, since Fig. 4a depicts the results for $G_{v}=0$, which means that no modifications to the provided nominal berth plan are allowed. Since the maximally required crane capacity reservation is scaled to the amount required for the case where $G_{v}=0$, and $U_{v}=4, \forall v$, indeed the values in Fig. 4a are equal to one for the case where $U_{v}=4$.

- As $G_{v}$ increases, the conventional berth plan typically requires a slightly smaller maximal crane capacity reservation than the window-based plan for small values of $U_{v}$. This is to be expected, since the conventional plan is based on zero disturbances and hence constructs an optimal deterministic plan. The window-based plan takes disturbances into account to construct a robust plan, which not necessarily has to be the optimal deterministic plan. The difference of the two graphs at $U_{v}=0$ therefore quantifies the price of robustness.

- As $G_{v}$ increases, the window-based plan requires a significantly smaller maximal crane capacity reservation than the conventional plan for medium and high values of $U_{v}$ that are still within the arrival windows. Apparently, the conventional plan is very sensitive to medium and large disturbances and it thus is worth taking the arrival agreements into account while constructing the berth plan. 
- If the nominal arrival times of the vessels in $\mathcal{V}_{2}$ are allowed to be modified maximally two shifts $(16 \mathrm{~h})$, the maximally required crane capacity reservation can be reduced by about $25 \%$ for $U_{v}=4$.

From Fig. 5, we conclude the following:

- In general, as the flexibility level $G_{v}$ increases, the maximal crane capacity reservation of the provided PSA HNN berth plan can be reduced for each value of $U_{v}$ by applying either the conventional or the window-based planning.

- The results for the conventional planning for the case where $U_{v}=0$ (Fig. 5a), and the results for the window-based planning for the case where $U_{v}=4$ (Fig. 5e) are monotonically decreasing. This makes sense, since the conventional plan and the window-based plan are typically based on arrival windows where $W_{v}=0$ and $W_{v}=4$, respectively. Hence, if the maximal plan modification $G_{v}$ increases, the maximal crane capacity reservation will never increase.

- All other curves in Fig. 5, however, are not necessarily monotonically decreasing. This is due to the fact that the conventional plan and window-based plan are constructed based on typical values of $W_{v}$ (see item above). If the actual arrival disturbances turn out to deviate from these window sizes $\left(U_{v} \neq W_{v}\right)$, an increase in $G_{v}$ might possibly increase the maximal crane capacity reservation.

\section{Conclusions}

We considered a planning problem of a terminal operator who has to construct a cyclic nominal timetable, according to which a set of cyclically arriving vessels is discharged and loaded. Disturbances on travel times, however, lead to stochastic arrivals in the port. To cope with these disturbances, the terminal operator and each of the vessel lines agree on a so-called arrival window placed around the nominal arrival times. Only if a vessel arrives within its window, the terminal operator has to process this vessel within the agreed nominal vessel process time. If a vessel arrives outside its window, the terminal operator is not bound to any process time.

We are interested in a possible reduction in the maximal crane capacity reservation by modifying (some of) the nominal arrival times in an existing berth plan. Furthermore, we want to explicitly take the arrival window agreements into account. An MILP is developed to construct a robust window-based cyclic berth plan with minimally required crane capacity in the worst case arrival scenario, i.e., an MILP that minimizes the maximally required crane capacity while the process agreements for all scenarios where vessels arrive within their windows are still met. Experiments on a nominal berth plan provided by the terminal operator PSA HNN in Antwerp, Belgium, show that with small modifications to the plan, already significant reductions in the maximal crane capacity reservation can be obtained.

Conventional berth planning methods ignore stochastic arrival behavior while constructing a nominal berth plan. As a consequence, actual deviations from the nominal arrival times may put a high pressure on quay and crane utilizations. As a particular case, our robust MILP model enables us to construct a conventional, deterministic plan by simply setting the arrival window width to zero. We investigated the performance of the window-based plan and the conventional plan for different levels of actual arrival 
disturbances. The results show that the window-based plan requires a slightly larger maximal crane capacity reservation than the conventional plan if actual arrivals turn out to be close to the constructed nominal arrival times. However, the window-based plan requires a significantly smaller maximal quay crane capacity reservation than the conventional plan if actual arrivals turn out to deviate more, but are still well within the arrival windows.

Negotiations have to reveal whether vessel lines are willing to modify their nominal arrival and departure times. The window-based model developed in this paper enables a terminal operator to quantify the spare crane capacity to be gained by these potential modifications.

Open Access This article is distributed under the terms of the Creative Commons Attribution Noncommercial License which permits any noncommercial use, distribution, and reproduction in any medium, provided the original author(s) and source are credited.

\section{References}

Ball M, Barnhart C, Nemhauser G, Odoni A (2007) Air transportation: irregular operations and control. In: Barnhart C, Laporte G (eds) Handbooks in operations research and management science: transportation, chap 1. Elsevier, Amsterdam, pp 1-67

Bertsimas D, Sim M (2004) The price of robustness. Oper Res 52(1):35-53

Caimi G, Fuchsberger M, Laumanns M, Schüpbach K (2007) Periodic railway timetabling with event flexibility. In: Liebchen C, Ahuja R, Mesa J (eds) ATMOS-7th workshop on algorithmic approaches for transportation modeling, optimization and systems. Internationales Begegnungs- und Forschungszentrum für Informatik (IBFI), Schloss Dagstuhl, Germany, pp 124-141

Clausen J (2007) Disruption management in passenger transportation-from air to tracks. In: Liebchen C, Ahuja R, Mesa J (eds) ATMOS - 7th workshop on algorithmic approaches for transportation modeling, optimization and systems. Internationales Begegnungs- und Forschungszentrum für Informatik (IBFI), Schloss Dagstuhl, Germany, pp 30-47

Cordeau JF, Laporte G, Legato P, Motta L (2005) Models and Tabu search heuristics for the berth-allocation problem. Transp Sci 39:526-538

Hansen P, Oğuz C, Mladenović N (2007) Variable neighborhood search for minimum cost berth allocation. Eur J Oper Res (in press)

Hendriks M (2009) Multi-step optimization of logistics networks. strategic, tactical and operational decisions. Ph.D. thesis, Eindhoven University of Technology

Imai A, Nishimura E, Papadimitriou S (2001) The dynamic berth allocation problem for a container port. Transp Res B 35:401-417

Imai A, Nishimura E, Papadimitriou S (2005) Berth allocation in a container port: using a continuous location space approach. Transp Res B 39:199-221

Imai A, Nishimura E, Hattori M, Papadimitriou S (2007) Berth allocation at indented berths for mega-containerships. Eur J Oper Res 179:579-593

Kim KW, Moon KC (2003) Berth scheduling by simulated annealing. Transp Res B 37:541-560

Lan S, Clarke J, Barnhart C (2006) Planning for robust airline operations: optimizing aircraft routings and flight departure times to minimize passenger disruptions. Transp Sci 40(1):15-28

Lim A (1998) The berth planning problem. Oper Res Lett 22:105-110

Moorthy R, Teo C (2006) Berth management in container terminal: the template design problem. OR Spectr 28(4):495-518

Nishimura E, Imai A, Papadimitriou S (2001) Berth allocation planning in the public berth system by genetic algorithms. Eur J Oper Res 131:282-292

Park YM, Kim KH (2003) A scheduling method for berth and quay cranes. OR Spectr 25:1-23

Vromans M, Dekker R, Kroon L (2007) Cyclic railway timetabling: a stochastic optimization approach. Lect Notes Comput Sci 4359:41-66

Wang F, Lim A (2007) A stochastic beam search for the berth allocation problem. Decis Support Syst 42:2186-2196 